\title{
Cosmology of the Nambu-Jona-Lasinio Model
}

\author{
Leonardo Quintanar G., A. de la Macorra \\ Instituto de Fisica, Universidad Nacional Autonoma de Mexico, A.P. 20-364, 01000, Mexico D.F., Mexico \\ Email: a.macorra@gmail.com
}

How to cite this paper: Leonardo Quintanar G. and de la Macorra, A (2016) Cosmology of the Nambu-Jona-Lasinio Model. Journal of Modern Physics, 7, 1777-1800. http://dx.doi.org/10.4236/jmp.2016.713159

Received: May 3, 2016

Accepted: September 27, 2016

Published: September 30, 2016

Copyright $\odot 2016$ by authors and Scientific Research Publishing Inc. This work is licensed under the Creative Commons Attribution International License (CC BY 4.0).

http://creativecommons.org/licenses/by/4.0/

\begin{abstract}
We review the Nambu and Jona-Lasinio model (NJL), proposed long time ago, in the sixties, as a fermion interaction theory with chiral symmetry. The theory is not renormalizable and presents a symmetry breaking due to quantum effects which depends on the strength of the coupling constant. We may associate a phase transition with this symmetry breaking, leading from fermion states to a fermion condensate which can be described effectively by a scalar field. Our purpose in this paper is to exploit the interesting properties of NJL in a different context other than particle physics by studying its cosmological dynamics. We are interested in finding whether possibly the NJL model could be used to describe the still unknown dark energy and/or dark matter, from up to $95 \%$ of the energy content of the universe at present time.
\end{abstract}

\section{Keywords}

Cosmology, Nambu Jona Lasinio

\section{Introduction}

In the last years the study of our universe has received a great deal of attention since, on the one hand fundamental theoretical cosmological questions remain unanswered and, on the other hand we have now the opportunity to measure the cosmological parameters with an extraordinary precision. In the last decades, research in cosmology has revealed the presence of unexplained forms of matter and energy called Dark Energy "DE" and Dark Matter "DM" making up to $95 \%$ of the energy content of the universe at present time. The study of supernovas SNIa shows that the universe is not only expanding, but besides it is accelerating [1]-[6]. Such behaviour can be explained by the existence of a new form of energy, Dark Energy with an anti-gravitational property, which would be explained by a fluid with negative pressure. Independent evidence for Dark Matter (DM) and Dark Energy (DE), is provided through the analysis of the 
Cosmic Microwave Background radiation (CMB) [7]-[10], which has been measured by satellite WMAP [11], and more recently by Planck mission [12], and the dynamics of galaxies, clusters and super clusters, and the study of the formation of Large Scale Structure [13]-[16] in the universe and weak lensing (the gravitational deviation of light), which point out the existence of matter that do interacts with ordinary standard model matter only weakly, as due to gravity. Other important measurements are the Baryon Acoustic Oscillations "BAO" [17]-[19].

It has been established that our universe is flat and dominated at present time by Dark Energy "DE" and Dark Matter "DM" with $\Omega_{D E} \simeq 0.692 \pm 0.02, \Omega_{m}=0.308 \pm 0.009$ and Hubble constant $H_{o}=(67.27 \pm 0.66) \mathrm{km} \cdot \mathrm{s}^{-1} \cdot \mathrm{Mpc}^{-1}$ [12]. However, the nature and dynamics of Dark Energy and Dark Matter are topics of major interest in the field [20]. The equation of state "EOS" of DE is at present time $w_{o} \simeq-0.93 \pm 0.13$ but we still do not have a precise measurement of $w(z)$ as a function of redshift $z$ [12] [16]. Since the properties of Dark Energy are still under investigation, different DE parametrizations have been proposed to help discern on the dynamics of DE [20]-[23]. Some of these DE parametrizations have the advantage of having a reduced number of parameters, but they may lack a physical motivation and may also be too restrictive. Perhaps the best physically motivated candidates for Dark Energy are scalar fields which can be minimally coupled, only via gravity, to other fluids [20]-[23] or can interact weakly in interacting Dark Energy "IDE” [24]-[27]. Scalar fields have been widely studied in the literature [20]-[23] and special interest was devoted to tracker fields [22] [23] since in this case the behavior of the scalar field $\phi$ is very weakly dependent on the initial conditions at a very early epoch and well before matter-radiation equality. In this class of models the fundamental question of why DE is relevant now, also called the coincidence problem, can be ameliorated by the insensitivity of the late time dynamics on the initial conditions of $\phi$.

Nowadays there are a huge number of ideas aimed to explain these unknown cosmological fluids DE and DM, from the theoretical point of view, none of them being still conclusive. This situation supports and motivates our research. Given that our most successful theory of matter, the Standard Model of particle physics (SM), which is settled within the theoretical frame of Quantum Field Theory (QFT), it would be reasonable to ask a theory attempting to describe dark fluids to be based on QFT as well. In this paper we study a fermion interaction theory with a chiral symmetry, the Nambu-Jona-Lasinio (NJL) model. Though this is an old and well known model in the context of hadron physics, it has interesting properties and it is worth to consider it with a different perspective, by studying its possible relevance for Cosmological Physics. Other examples of QFT models of DE and DM have been proposed using gauge groups, similar to QCD in particle physics, and have been studied to understand the nature of Dark Energy [28] [29] and also Dark Matter [30] [31].

We organized the present work as follows: In Section 2 we present the NJL model. In Section 3 we review the pertinent cosmological theory. Sections 4 and 5 present a study of the cosmological dynamics of a NJL fluid with a weak and strong coupling, respec- 
tively. In Section 6 we consider the addition of a cosmological constant to our NJL fluid, and analyze the different possible behaviours. In Section 7 we comment an interesting possible way to modify the original NJL model, obtaining an additional term in the effective potential which could be related with a Cosmological Constant. Finally, in Section 8 we summarize our results and present the conclusions.

\section{The Nambu-Jona-Lasinio Model}

Inspired by a, by then recently explained phenomenon in Superconductivity research, professors Y. Nambu and Jona-Lasinio, suggested that the mass of fermion particles (described by a Dirac equation) could be generated from a primary four-fermion self interaction, leading to a chiral symmetry breaking. The proposed Lagrangian, invariant under chiral transformations, has the form

$$
\mathcal{L}=i \bar{\psi} \gamma^{\mu} \partial_{\mu} \psi+\frac{g^{2}}{2}\left[(\bar{\psi} \psi)^{2}-\left(\bar{\psi} \gamma_{5} \psi\right)^{2}\right],
$$

where $\psi$ is a four-component spinor, and $g$ is a coupling constant. From Equation (1) the four fermion interaction term is given by

$$
\mathcal{L}_{\text {int }}=\frac{g^{2}}{2}\left[(\bar{\psi} \psi)^{2}-\left(\bar{\psi} \gamma_{5} \psi\right)^{2}\right]
$$

with no original mass term for the fermions. Since the coupling has dimension-2 in mass units, the theory is non-renormalizable. However, we are interested in considering the NJL model as an effective theory, useful below certain energy scale. The theory (1) describes a four-fermion interaction which can be expanded following conventional perturbation theory, and represented by Feynman diagrams (Figure 1).

The infinite number of fermion loops can be resumed giving a non-perturbative potential. This can be easily done by introducing an auxiliary scalar field $\phi$ and an equivalent Lagrangian for Equation (2) in the form

$$
\mathcal{L}_{\text {int }}=m g \varphi \bar{\psi} \psi-\frac{1}{2} m^{2} \phi^{2} .
$$

The field $\phi$ plays the role of a Lagrange multiplier which can be eliminated using the Euler-Lagrange equations, $\partial_{\mu} \frac{\delta \mathcal{L}}{\delta\left(\partial_{\mu} \phi\right)}-\frac{\delta \mathcal{L}}{\delta \phi}=0$. For the Lagrangian above we find

$$
\phi=\frac{g}{m} \bar{\psi} \psi,
$$

where $\phi$ has mass dimensions, and by substituting Equation (4) in Equation (3) one can recover the original Lagrangian Equation (2). Note that we introduced the parame-

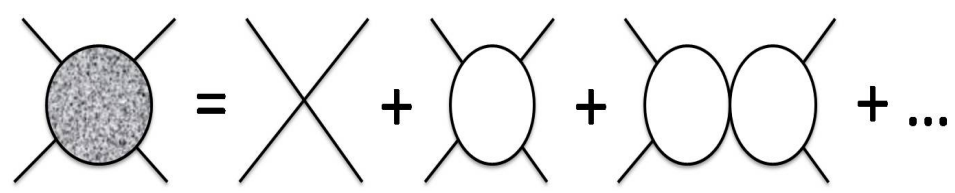

Figure 1. Feynman diagram for a four-fermion interaction. 
ter $m$ with a physical dimension of mass, so that $m g$ is a dimensionless coefficient, and we have dimensional consistency for all the physical quantities. ${ }^{1}$ The term $\bar{\psi} \gamma_{5} \psi$ in Equation (1) represents a pseudo-scalar quantity, and we have allowed ourselves to ignore the field contribution associated with it in the new Lagrangian in Equation (3), as we would like to start to study the simplest possible model. ${ }^{2}$

From the equivalent Lagrangian one may read the fermion mass and the tree level scalar potential $V_{0}$. We have respectively:

$$
m_{\psi}^{2}=(m g \phi)^{2}, \quad V_{0}=\frac{1}{2} m^{2} \phi^{2} .
$$

The effect of quantum processes (represented by loop diagrams) may be taken into account through the well known Coleman-Weinberg potential

$$
V_{1}=-\frac{1}{8 \pi} \int p^{2} \log \left(p^{2}+m_{\psi}^{2}\right) \mathrm{d}^{2} p,
$$

the minus sign in $V_{1}$ is because it corresponds to the fermionic contribution to the Coleman-Weinberg potential. As we will see, for the strong coupling case, this will enable the effective potential to adopt a negative value when the field stabilizes at the minimum. The integral grows up indefinitely as the upper limit goes to infinity, i.e. it has an ultraviolet divergence. Because of the non-renormalizability of the theory, we cannot avoid this divergence, so we regularize by introducing a cut-off $\Lambda$. This parameter defines the energy scale below of which the theory is valid. We define the $x$ variable as

$$
x \equiv \frac{m_{\psi}^{2}}{\Lambda^{2}}=\frac{m^{2} g^{2} \phi^{2}}{\Lambda^{2}}
$$

and the potential becomes

$$
\begin{gathered}
V_{0}=\frac{\Lambda^{2} x}{2 g^{2}} \\
V_{1}=-\frac{\Lambda^{4}}{16 \pi^{2}}\left[x+x^{2} \log \left(\frac{x}{1+x}\right)+\log (1+x)\right] .
\end{gathered}
$$

Notice that the one-loop potential $V_{1}$ is negative since it corresponds to the contribution of the original fermion field $\psi$, and we choose to parameterize it in terms of the effective scalar field $\phi$ c.f. Equation (4).

For the sake of concision we also define

$$
A \equiv \frac{\Lambda^{4}}{16 \pi^{2}}, \quad f(x)=x+x^{2} \log \left(\frac{x}{1+x}\right)+\log (1+x) .
$$

In this way, taking quantum corrections into account we obtain an effective potential given by

$$
V=V_{0}+V_{1}=\frac{\Lambda^{2} x}{2 g^{2}}-A f(x),
$$

${ }^{1}$ Remember that the dimension of a scalar field equals that of mass.

${ }^{2}$ It will become clear that by doing so does not affect qualitatively the implied physical processes. 
with the complete potential

$$
V=V_{0}+V_{1}=\frac{\Lambda^{2} x}{2 g^{2}}\left(1-\frac{g^{2} \Lambda^{2}}{8 \pi^{2}}\right)-\frac{\Lambda^{4}}{16 \pi^{2}}\left[x^{2} \log \left(\frac{x}{1+x}\right)+\log (1+x)\right] .
$$

As a function of $\phi$ it can be written explicitly as

$$
V(\phi)=\frac{1}{2} m^{2} \phi^{2}-\frac{\Lambda^{4}}{16 \pi^{2}}\left[\left(\frac{m g \phi}{\Lambda}\right)^{2}+\left(\frac{m g \phi}{\Lambda}\right)^{4} \log \left(\frac{\left(\frac{m g \phi}{\Lambda}\right)^{2}}{1+\left(\frac{m g \phi}{\Lambda}\right)^{2}}\right)+\log \left(1+\left(\frac{m g \phi}{\Lambda}\right)^{2}\right)\right] \text {. }
$$

Equation (13) gives the complete NJL scalar potential, and we are interested in studying its cosmological implications. Let us determine the asymptotic behaviour of the scalar potential $V$ in Equation (13). To analyze the potential we seek for extremum points. For the function $f(x)$ in Equation (10) we have the derivative

$$
\frac{\mathrm{d} f(x)}{\mathrm{d} x}=2\left[1+x \log \left(\frac{x}{1+x}\right)\right],
$$

and for the derivative of $V$ we have

$$
\begin{aligned}
& \frac{\partial V}{\partial \phi}=\frac{m^{2} \Lambda^{2} \phi}{4 \pi^{2}}\left\{\frac{4 \pi^{2}}{g^{2} \Lambda^{2}}-1-\left(\frac{m g \phi}{\Lambda}\right)^{2} \log \left(\frac{\left(\frac{m g \phi}{\Lambda}\right)^{2}}{1+\left(\frac{m g \phi}{\Lambda}\right)^{2}}\right)\right\}, \\
& \frac{\partial V}{\partial \phi}=\frac{m^{2} \Lambda^{2} \phi}{4 \pi^{2}}\left\{\frac{4 \pi^{2}}{g^{2} \Lambda^{2}}-1-x \log \left(\frac{x}{1+x}\right)\right\} .
\end{aligned}
$$

The condition $\frac{\partial V}{\partial \phi}=0$ implies the following equations:

$$
\text { i) } \phi=0, \quad \text { or } \quad \text { ii) } \frac{4 \pi^{2}}{g^{2} \Lambda^{2}}-1=x \log \left(\frac{x}{1+x}\right) \text {. }
$$

The first one says that the origin $\phi=0$ is an extremum, and if we take the second derivative $\frac{\partial^{2} V}{\partial \phi^{2}}$

$$
\left.\frac{\partial^{2} V}{\partial \phi^{2}}\right|_{\phi=0}=\frac{m^{2} g^{2} \Lambda^{2}}{4 \pi^{2}}\left(\frac{4 \pi^{2}}{g^{2} \Lambda^{2}}-1\right),
$$

we see that if $\frac{4 \pi^{2}}{g^{2} \Lambda^{2}}>1$ then the extremum at $\phi=0$ corresponds to a minimum, while for $\frac{4 \pi^{2}}{g^{2} \Lambda^{2}}<1$ we have a maximum at the origin. The equation above suggest to define a critical value of the coupling $g_{c}$ as

$$
g_{c}^{2} \equiv \frac{4 \pi^{2}}{\Lambda^{2}}
$$

so that we see that for a weak coupling $g<g_{c}$ we have a minimum at the origin, while 
at strong coupling $g>g_{c}$ we have a maximum. The type of extrema at the origin of the potential corresponds to the value of the coupling.

Now let us determine the second (possible) extreme of the potential. Since the r.h.s of the second equation in Equation (17) is negative (i.e. $\left.x \log \left(\frac{x}{1+x}\right) \leq 0\right)$ this equation has a solution only for a strong coupling $g>g_{c}$. A value for $x$ (or that of the scalar field $\phi$ ), at the minimum cannot be solved analytically, since the second equation in Equation (17) is a transcendental equation. One way to determine a solution is to seek for the intersection between the curve of the function $x \log \left(\frac{x}{1+x}\right)$ r.h.s. in the second Equation (17), and the constant in the 1.h.s. In this case do exist an intersection (only one, as the r.h.s. is a monotonic function), giving a solution for the $x$ variable, leading in its turn to a non-trivial solution in $\phi=\phi_{\min }$ which is a minimum. ${ }^{3}$ The extremum in this case corresponds to a minimum. Notice that in all cases we have at large $x$ the limit $V \rightarrow \infty$ for $x \rightarrow \infty$ regardless of the value of the coupling $g$.

Therefore, we have: if $g<g_{c}$, the potential minimizes in the origin $\phi=0$; whereas for $g>g_{c}$, the potential minimizes in a non trivial value $\phi=\phi_{\min }$. The value of the coupling $g=g_{c}$ define a critical value separating between both behaviours of the potential (in Figure 2 we show all the three cases $\left.g<g_{c}, g=g_{c}, g\right\rangle g_{c}$ ). When for $g>g_{c}$ we see that the full potential $V=V_{0}+V_{1}$ becomes negative, due to the contribution of $V_{1}$, and a fermion condensate $\bar{\psi} \psi \neq 0$ is formed and is parameterized by the scalar field $\phi=(\mathrm{g} / \mathrm{m}) \bar{\psi} \psi$, c.f. Equation (4).

To estimate the value of the potential at the minimum for $g>g_{c}$, the equation ii) in Equation (17) should be solved. However, since it is a transcendental equation in the variable $x$, an algebraic expression cannot be written, and we need to use numerical

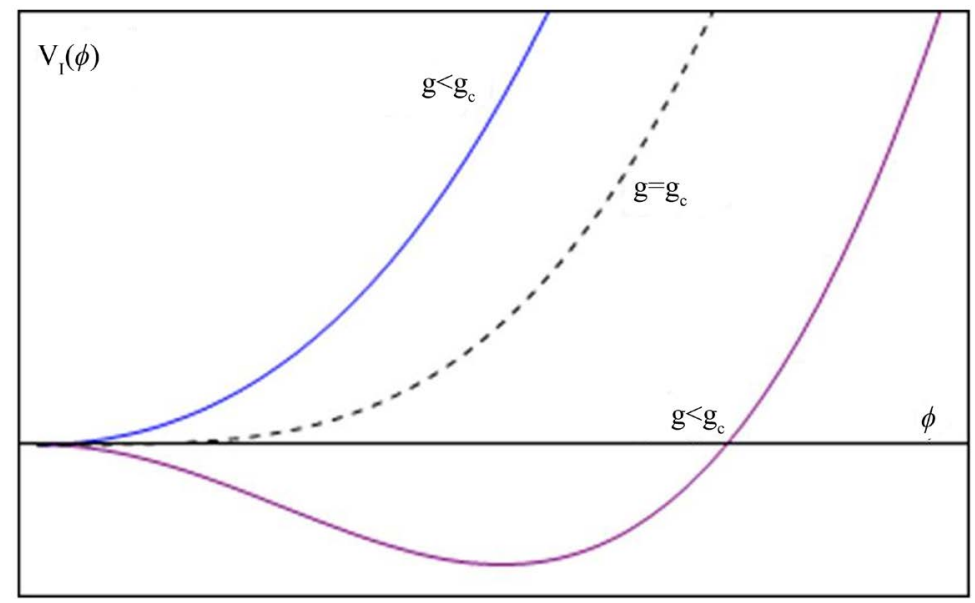

Figure 2. Effective potential (13) as a function of $\phi$. The critical value of the coupling $g_{c}$, separates two kinds of behaviours.

\footnotetext{
${ }^{3}$ According to definition Equation (7), $x$ is a quadratic function in $\phi: x \sim \phi^{2}$, so for a given value of $x$ we have two solutions in $\phi$ related by a change of sign. Due to this symmetry, we will allow ourselves to refer to only one solution.
} 
procedures. Let us introduce a parameter $s$ to write $g$ in the form

$$
g=s \cdot g_{c}=s \cdot \frac{2 \pi}{\Lambda} \text {. }
$$

In this way we make sure to have a strong coupling by taking $s>1$. Now, for a given value of the coupling with $g>g_{c}$, there exists a definite value of $x$, say $x_{0}$, satisfying the condition ii) in Equation (17), which is the solution for the minimum. Therefore, the potential valuated at this point yields the minimized potential, i.e. $V_{\min }=V\left(x_{0}\right)$. Then, by substituting ii) Equation (17), and using Equation (20) in the expression for the potential Equation (12), we can write $V_{\min }$ in the suitable form

$$
V_{\min }=\frac{\Lambda^{4}}{16 \pi^{2}}\left[\frac{x_{0}}{s^{2}}-\log \left(1+x_{0}\right)\right], \quad\left(g=s \cdot g_{c}, s>1\right),
$$

which provides a good idea of how $V_{\min }$ is related to the energy scale.

From Equation (4) the field $\phi \sim \bar{\psi} \psi$, is a Lorentz invariant quantity, so $\phi$ is scalar field. When the field $\phi$ is stabilized, a non trivial expectation value reflects the presence of a fermion condensate.

Now, if the field has an expectation value $\langle\phi\rangle=0$, it means that the state of paired fermions $\bar{\psi} \psi$ is not present, so we have a system consisting in the original massless fermion particles with a 4-Fermi interaction, and a condensate is not energetically favoured. This happens for a "weak" coupling $g<g_{c}$. On the other hand, if the expectation value $\langle\phi\rangle \neq 0$, then we have a fermion condensate represented effectively by the scalar field. This happens for a "strong" coupling $g>g_{c}$, and a fermion condensate is dynamically formed since it reduces the energy of the system.

Thus, we see that two different fluid phases (massless fermions or fermion condensate) are obtained depending on the strength of the coupling. Next, we investigate the cosmological dynamics of each of these fluids.

\section{Standard Cosmology}

The widely accepted current standard cosmological model (the Big Bang theory) is based in Einstein's theory of General Relativity. If conditions of spatial homogeneity and isotropy are assumed, the space-time metric adopt the well-known simple form

$$
\mathrm{d} s^{2}=\mathrm{d} t^{2}-a(t)^{2}\left[\frac{\mathrm{d} r^{2}}{1-k r^{2}}+r^{2} \mathrm{~d} \theta^{2}+r^{2} \sin ^{2} \theta \mathrm{d} \phi^{2}\right]
$$

where the variables $r, \theta, \phi$ are comoving coordinates parameterizing the spatial section of space-time, and $k$ takes the values $+1,0,-1$ for spaces of constant positive (spherical), zero (flat), or negative (hyperbolic) curvature. When this metric is used in the Einstein's equations, the so called FRWL equations (Friedmann-Robertson-WalkerLemaitre) can be obtained. As these assumptions agree with observations $s^{4}$ to a very high precision, we will use this same theoretical framework. Because the necessary equations are well known and their deduction can be found in standard text books, in the 
following we limit ourselves to write them and to give only a brief explanation.

The equation

$$
\frac{\dot{a}^{2}}{a^{2}}+\frac{k}{a^{2}}=\frac{8 \pi G}{3} \rho,
$$

relates the expansion rate (in time) of the scale factor $a$, and the curvature $k$ of the universe, to the total energy density $\rho$. Along this paper we will always take a flat geometry $k=0$, as suggested on the one hand from the theory of early cosmological inflation, and on the other hand (and most important) from observation of the CMBR.

Introducing the usual definition relating the Hubble parameter $H$ with the rate of change in time of the scale factor $a$

$$
\dot{a}=a \cdot H,
$$

Equation (23) (with $k=0$, flat universe) becomes

$$
H^{2}=\frac{8 \pi G}{3} \rho .
$$

The continuity equation for a fluid with energy density $\rho$ and pressure $P$ is

$$
\dot{\rho}+3 H(\rho+P)=0 .
$$

For a perfect fluid " $\alpha$ " satisfying a barotropic equation of state $P_{\alpha}=w_{\alpha} \rho_{\alpha}$, with $w_{\alpha}$ a constant, Equation (26) can be solved analytically. We sometimes will refer to such a fluid with the name of "barotropic fluid". From the cosmological point of view, the substances contained in the universe can be described as radiation, which has $w_{r}=1 / 3$, and matter (dust) having $w_{m}=0$ (besides the Dark Energy component). For those we have respectively

$$
\rho_{r}=\rho_{r i}\left(\frac{a}{a_{i}}\right)^{-4}, \quad \rho_{m}=\rho_{m i}\left(\frac{a}{a_{i}}\right)^{-3} .
$$

A scalar field $\phi$, with a self-interaction potential $V(\phi)$, has energy density $\rho_{\phi}$ and pressure $P_{\phi}$ given by

$$
\rho_{\phi}=E_{k}+V(\phi), \quad P_{\phi}=E_{k}-V(\phi), \quad E_{k}=\frac{1}{2} \dot{\phi}^{2}
$$

where we have also defined the kinetic energy $E_{k}$ in the third equation. Considering an universe containing radiation, matter and a scalar field, the total energy density is written

$$
\rho=\rho_{r}+\rho_{m}+\rho_{\varphi} .
$$

For a given component fluid " $\alpha$ ", it is useful to know its relative density, defined as the ratio of its energy density to the total energy density:

$$
\Omega_{\alpha}=\frac{\rho_{\alpha}}{\rho}=\frac{8 \pi G \rho_{\alpha}}{3 H^{2}},
$$

where we have used Equation (25) in the second equality. In a flat universe one has the condition

$$
\Omega_{r}+\Omega_{m}+\Omega_{\phi}=1
$$


It is interesting to note that while Equation (31) remains valid even when we have a negative $\rho_{\alpha}$, the quantity $\Omega_{\alpha}$ is no longer constrained to the values $0 \leq \Omega_{\alpha} \leq 1$. In the work presented here, the fluids can have a negative energy density, giving $\Omega_{\alpha}<0$, or a total energy density $\rho$ that vanish at finite values of the scale factor $a(t)$, in which case we would have $\Omega_{\alpha} \rightarrow \pm \infty$.

Taking the time derivative in Equation (25) and using Equation (26), it can be found

$$
\dot{H}=-\frac{1}{2}\left(\rho_{m}+\frac{4}{3} \rho_{r}+\dot{\phi}^{2}\right) \text {. }
$$

Note that the r.h.s. in Equation (32) is always negative. The equation of motion for a spatially homogeneous scalar field, (a modified Klein-Gordon equation) is given by

$$
\ddot{\phi}+3 H \dot{\phi}+\partial_{\phi} V=0 .
$$

It is also useful an equation for the acceleration of the scale factor:

$$
\frac{\ddot{a}}{a}=-\frac{4 \pi G}{3}(\rho+3 P) .
$$

Differential Equations (24), (32), (33), together with (27) constitute a complete set which can be solved numerically (since we cannot always write an analytical solution). Nevertheless, it is convenient to attempt to outline the general behaviour of the dynamical system. Thus, before going to solve for our NJL potential, let us point out the following generic facts:

The evolution of the scalar field is such that it will minimize the scalar potential $V(\phi)$, so for an arbitrary initial value $\phi_{i}$, the field will roll to lower values of the potential, in such a way that eventually it will adopt a constant value $\left(\phi=\phi_{\min }\right.$ being the minimum). Given than the scale factor is a positive defined quantity, the energy densities for matter and radiation Equation (27) are always positive quantities and never equal to zero for finite values of the scale factor $a(t)$. So, the total energy density Equation (29) remains always positive as long as the condition

$$
\rho=\rho_{r}+\rho_{m}+\rho_{\phi}>0
$$

is satisfied. Thus, Equation (25) says that $H=0$, that is $\dot{a}=0$, never happens (Equation (24)) as long as $\rho \neq 0$. This implicates that $\dot{a}>0$ always. This means that the scale factor $a(t)$ never reaches an extremum value along its time evolution (taking an initial condition $H_{i}>0$, since we know that the universe is expanding at present time).

Nevertheless, it is interesting o note that there is no known physical principle forbidding the existence of a fluid with a negative potential $V(\phi)<0$, at least for some values of the field $\phi$. In this case, it could well happen that Equation (35) become an equality, meaning $\rho=0$ for finite values of $a(t)$, which in turn implies $H=0$, and $\dot{a}=0$; i.e., the scale factor reaches an extremum value (indeed a maximum, since as seen before, it was initial growing). Now, Equation (32) imposes an always decreasing Hubble parameter $H$ (because the right hand side is always negative), so that after being $H=0$ it must be $H<0$, and therefore $\dot{a}<0$, i.e. the scale factor decrease. In other 
words, the universe must be contracting after reaching its maximum size. Observe that this result is a consequence only of the negativity of the potential, and it is independent of its specific form. This collapsing universe is valid even for a flat universe $k=0$. To conclude, if a fermion condensate is energetically favored then the minimum of potential $V(\phi)$ is negative and the universe will recolapse.

\section{Dynamics of Massless Fermions Phase (Weak Coupling $g<g_{c}$ )}

As we have seen in Section 2, for a weak coupling $g<g_{c}$ the minimum of the potential $V\left(\phi_{\min }\right)=0$ is located at the origin with $\phi_{\min }=0$, and $V$ does not take negative values. Therefore, the total energy density and $H$ never vanish for finite values of the scale factor $a$, and we have $\dot{a}>0$ due to Equation (24). So the scale factor $a(t)$ is always growing, going to an infinite size in an infinite time. Now, from Equation (34), it can be seen that, in order to have $\ddot{a}<0$, i.e. the universe to slow down its expansion rate, then

$$
\frac{2}{3} \rho_{r}+\frac{1}{2} \rho_{m}+\dot{\phi}^{2}>V(\phi)
$$

is a condition to be satisfied. This, of course, in not always the case: we could take an initial field amplitude $\phi_{i}$ as big to make the initial value of the potential $V_{i}=V\left(\phi_{i}\right)$ big enough so that inequality (36) does not hold, and we would have instead $V>\frac{2}{3} \rho_{r}+\frac{1}{2} \rho_{m}+\dot{\phi}^{2}$. In this case we could have an acceleration of the scale factor, i.e. an accelerating universe, though it would be an "early" acceleration, as it would be present an initial times, i.e. before letting the fluid densities to dilute and field to evolve. As time passes, the field rolls down minimizing the potential, and eventually acquires some value $\phi<\phi_{i}$ such that condition (36) becomes fulfilled.

Given that the densities of matter and radiation never reach a null value in a finite time, and that the field amplitude tends to be stabilized around the minimum (i.e. $\phi \rightarrow 0$ ), for a big enough amount of time, we expect a vanishing potential and velocity, $V \sim 0, \dot{\phi} \sim 0$ to be a good approximation to a final situation, in which (36) is still satisfied.

We show an example of numerical solution in the figures. In Figure 3 we see that the field has a damped oscillation around $\phi=0$, and in consistency with this, its kinetic energy (velocity) diminish in time and we show in Figure 4 the evolution of the relative densities $\Omega_{\text {rad }}, \Omega_{\text {mat }}, \Omega_{\phi}$ for radiation, matter and $\phi$. Simultaneously, the potential valuated at $\phi \sim 0$ goes to lower values (according to $V\left(\phi_{\min }\right)=0$ ). We can see that although the universe is expanding, it always ends up in a non-accelerating regime (Figure 5). A Taylor expansion for the potential about $\phi=0$ gives

$$
V \simeq \frac{1}{2} m^{2}\left(1-\frac{\Lambda^{2} g^{2}}{4 \pi^{2}}\right) \phi^{2},
$$

where the whole coefficient multiplying on $\phi^{2}$, is a positive quantity, as $g^{2}<4 \pi^{2} / \Lambda^{2}$. The coefficient of state $\omega_{\phi}$ defined below Equation (26), for the field $\phi$, written explicitly is 

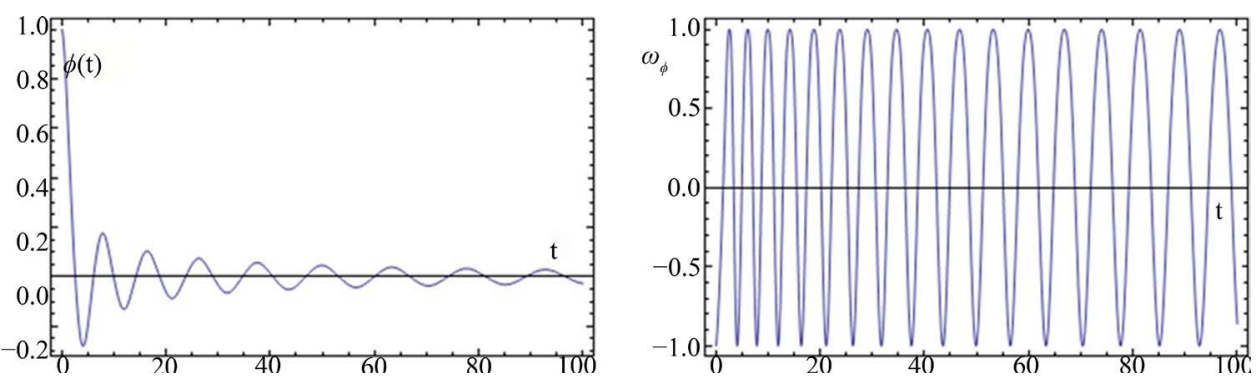

Figure 3. Left: Scalar field amplitude $\phi$. Right: State equation coefficient $\omega_{\phi}$. Both variables are shown as functions of time.
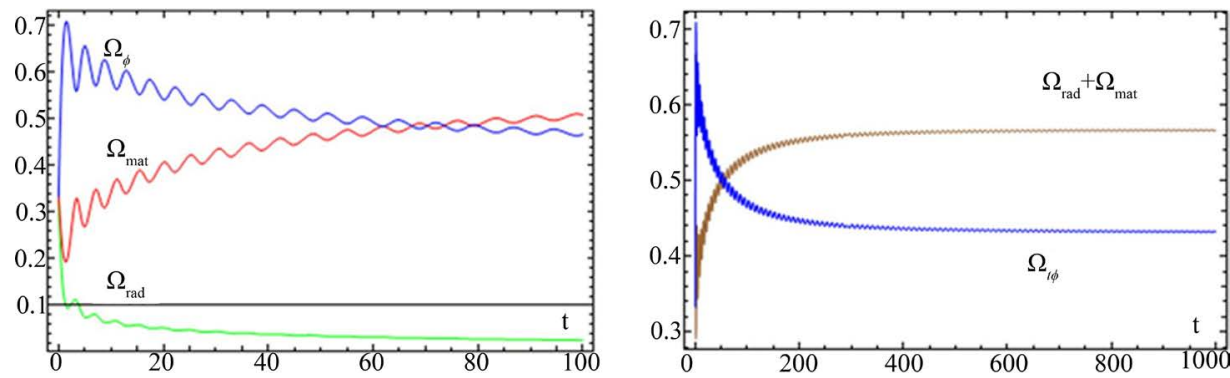

Figure 4. Left. Relative densities $\Omega_{\alpha}$ for radiation, matter and $\phi$. Right. Total relative density for barotropic fluids (matter and radiation) and for the field $\phi$. The horizontal axis in both graphics represents time. Note that we show a different scale of time in each plot for the same solution.
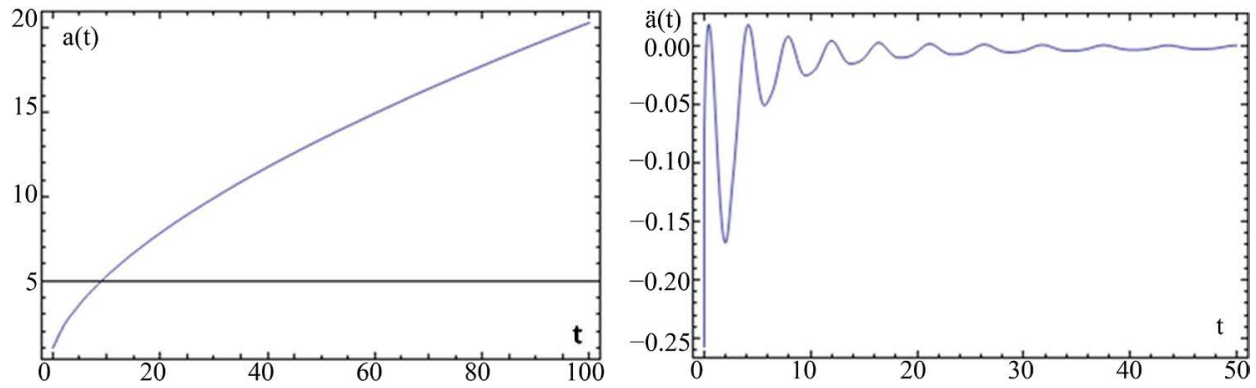

Figure 5. Left: Scale factor $a(t)$. Right: Acceleration of the scale factor $\ddot{a}(t)$. Both variables are shown as functions of time. Note that $\ddot{a}(t)$ adopt mostly negative values (tends to zero from below).

$$
\omega_{\phi}=\frac{P_{\phi}}{\rho_{\phi}}=\frac{E_{k}-V}{E_{k}+V} .
$$

Since at late times, when the field oscillates around its minimum with a quadratic potential, the average value is $\left\langle\omega_{\phi}\right\rangle=0$ and $\rho_{\phi}$ evolves as matter with $\rho_{\phi} \propto a^{-3}$ [24].

Within the context of Early Cosmic Inflation theory, the so called Slow Roll parameters are defined as follows:

$$
\epsilon=\frac{M_{p}^{2}}{2}\left(\frac{V^{\prime}}{V}\right)^{2}, \quad \eta=M_{p}^{2}\left(\frac{V^{\prime \prime}}{V}\right),
$$


which have to satisfy the conditions $\epsilon<1,|\eta|<1$ in order to the potential may cause a positive acceleration. Even though they are valid for a single field, without additional fluids (matter and/or radiation), we show them in Figure 6 the Slow Roll parameters, for the seek of completeness.

\section{Fermions Condensate Dynamics (Strong Coupling, $g>g_{c}$ )}

The strong coupling case leads to a fermion condensate and therefore to a negative potential $V$ at its minimum. The potential has at the origin $V(\phi=0)=0$ and decreases to negative values for $0<\phi<\phi_{\min }$. For $\phi>\phi_{\min }$ it grows monotonically, eventually passing from negative to positive values. Let us consider at first the simpler approach of a universe containing only a scalar field ( $\rho_{r}=\rho_{m}=0$, i.e. no additional fluids), evolving under a generic potential possessing a negative value $V_{\min }=V\left(\phi_{\min }\right)<0$ when minimized. If the initial velocity $\dot{\phi}_{i}=0$, then the kinetic energy of the field has a null value as well, so we have for the initial energy density $\rho_{\phi_{i}}=V_{i}$. The initial amplitude for the field $\phi_{i}$ cannot be such that makes $V\left(\phi_{i}\right)<0$, because it would lead to an imaginary value for $H$, according to Equation (25). Thus, we must take always $\phi_{i}$ such that $V_{i}>0$. As before we begin with $H_{i}=1>0$, therefore Equation (24) says that $a(t)$ initially is increasing in time. The equation (32) is written $\dot{H}=-(1 / 2) \dot{\phi}^{2}$, so that $H$ always diminish in time. As the potential is minimized, it goes from positive to negative values, and from Equation (25) eventually it will be $H=0$, and after this $H<0$, corresponding respectively to $\dot{a}=0$ and $\dot{a}<0$. In words this means that after an initial period of expansion (increasing scale factor), a maximum value is reached, followed by a period of contraction. Since $\dot{H}$ remains always negative, then $a(t)$ will continue decreasing, so that it necessarily will collapse. In other words, it will be $a=0$ in a finite time in the future (because the evolution is forward in time: the field minimizes, not otherwise).

Now, while the expanding phase is taking place, the field is rolling down, eventually entering in a damped oscillatory regime nearly the minimum, where the potential has become negative, $V_{\min }<0$. Because of the damping, the kinetic energy tends to a zero value, $E_{k} \rightarrow 0$. Thus, the energy density of the field $\rho_{\phi}=E_{k}+V$ goes from positive values (near $\phi_{i}$ ) to negative values (near $\phi_{\min }$ ), so at some time in between, it is
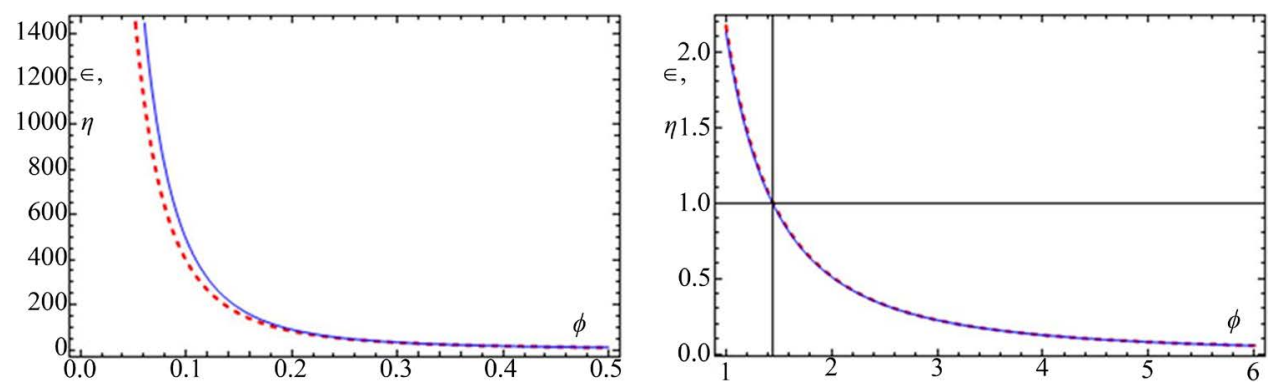

Figure 6. Slow roll parameters $\epsilon$ (dashed-red curve), and $\eta$ (continuous-blue). Left. From $\phi=0$ to $\phi=0.5$. Right. From $\phi=1$ to $\phi=6$. Only in the region $\phi \gtrsim 1.4$ approx. (and further on) one can expect the acceleration conditions $\epsilon<1, \eta<1$ to be satisfied. 
$\rho_{\phi}=0$. The total energy density, as well as the individual densities for each fluid (if there were additional fluids), would go to diminish in time (as can be seen for radiation and matter in Equation (27) with $\rho_{\alpha} \sim a^{-n}$, and $a(t)$ increasing). By a similar reasoning, because $a(t)$ is decreasing in the contracting phase, the energy densities behave the opposite way, i.e. they all increase in time. Therefore, we expect $\rho_{\phi}=0$ to happen twice. In its turn, this implicate that the coefficient of state $\omega_{\phi}$, Equation (38) become a divergent quantity also twice, around this two points, and near them, $\omega_{\phi}$ is not anymore a useful parameter to characterize the fluid represented by the field $\phi$. Below we show a numerical solution example (Figures 7-11).

As we mention before, in Section 3, a similar circumstance arises in dealing with the relative densities $\Omega_{\alpha}$ : it is considered that in order to this parameter to make sense, a relative density should adopt values $0 \leq \Omega_{\alpha} \leq 1$. However, as can be seen in Equation (30), if at some time is $H=0$, then nearly this value, each $\Omega_{\alpha}$ turns into a divergent variable. The situation is even weirdest for the field, because near the minimum it is $\rho_{\phi} \sim V_{\min }<0$, the energy density of the field is similar to the potential, which is negative. This would make $\Omega_{\alpha} \rightarrow-\infty$ (a divergent and negative relative density!).

Consider now a universe containing matter and radiation in addition to our NJL fluid. An interesting question is, may the presence of these fluids prevent the universe to collapse? Remember that the condition for an increasing scale factor can be reduced to the inequality (35). If the scale factor is supposed to grow forever, this condition must be hold always. Now, according to the explanations given above, initially the scale factor is growing indeed. Thus, from Equation (27) we see that the densities of both barotropic fluids (matter and radiation) must be decreasing. At the same time, because the field is stabilizing in the minimum of the potential, the kinetic energy of the field $E_{k}=(1 / 2) \dot{\phi}^{2}$ is diminishing to zero, whereas the potential is going to a constant value $V \rightarrow V_{\text {min }}$, in such a way that necessarily, condition (35) ceases to hold. Therefore, even in presence of additional barotropic fluids (does not matter the relative amount with respect to that of the fluid associated with the field), the collapsing universe situation cannot be avoided.

The previous qualitative generic analysis is verified by the numerical solution for our NJL potential in particular (Figures 7-11). By observing the graphics, we found an unpredicted interesting non-trivial behaviour of the field amplitude: while the scale factor undergoes the expanding, and contracting phases successively, an damped oscillating phase around $\phi_{\min }$ is taking place, as expected. But then, at some point in the contracting phase, the field amplitude goes to bigger values, and as the scale factor approach to $a=0$, the field is taken out from the minimum and it begins to increase monotonically! ${ }^{5}$ Is this an acceptable result? Intuitively, as a is decreasing, it is reasonable to expect all densities to be growing. In particular, if the field density $\rho_{\phi}=E_{k}+V$ is getting bigger, it should be due to an increase in the field velocity (so $E_{k}$ gets bigger), or in the field amplitude (so $V$ gets bigger); or both. This behaviour can indeed be

${ }^{5}$ It could decrease instead, depending on the initial conditions. Whatever the case, the monotonic growing in absolute value is an unexpected behaviour, which do happen indeed. 

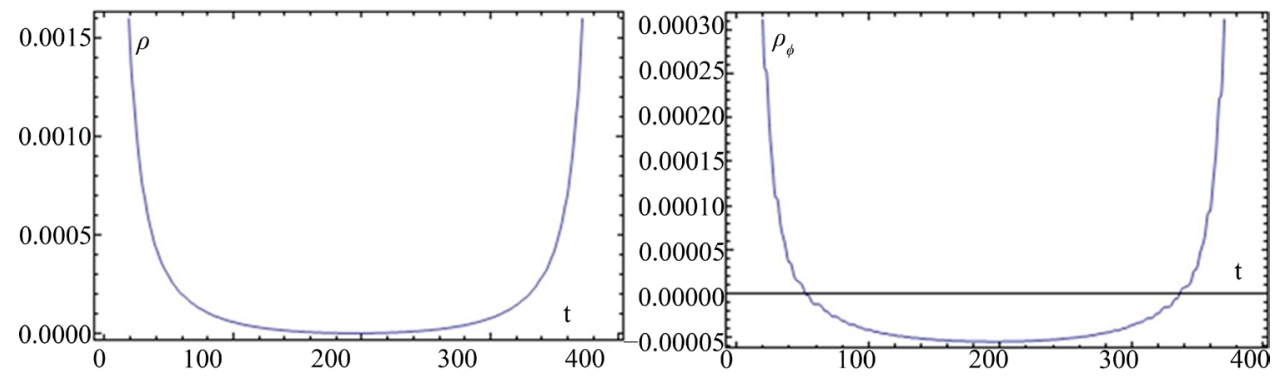

Figure 7. Left: Total energy density $\rho=\rho_{r}+\rho_{m}+\rho_{\phi}$. It is a positive quantity, but vanishes at a single point, near $t \simeq 200$ approx. Right. Energy density of the field. It is a null quantity $\left(\rho_{\phi}=0\right)$ twice: one time in the expansion phase (near $t=60$ approx.), and again in the contraction phase (about $t=340$ approx.); and becomes a negative quantity in between.
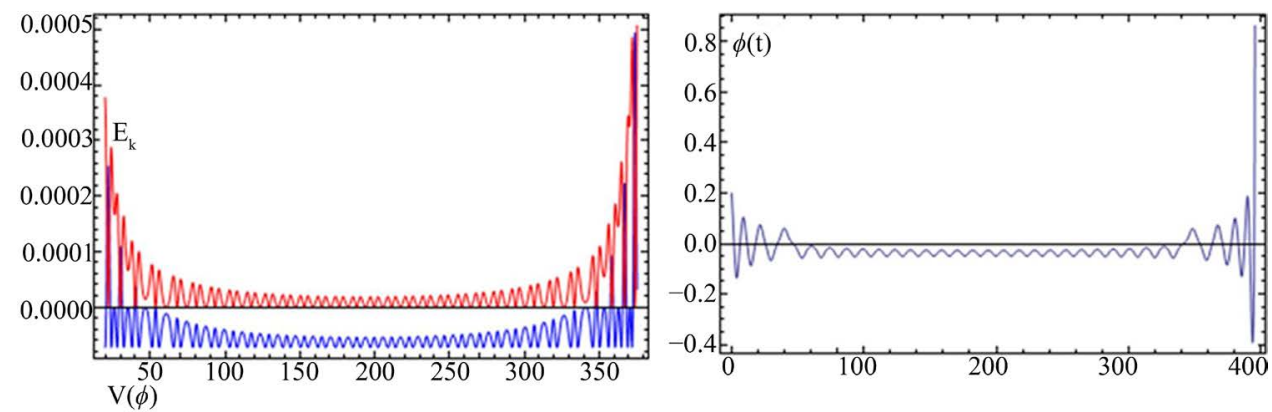

Figure 8. Left. Although the kinetic energy (red-upper curve) is zero initially, it overtakes the potential energy (blue-lower curve) and remains dominant all the way even to the collapsing time when $a(t)=0$. Right. The field oscillates around $\phi_{\min }$ and is becoming divergent as getting close to $t \simeq 400$, which is the time when $a(t) \rightarrow 0$.
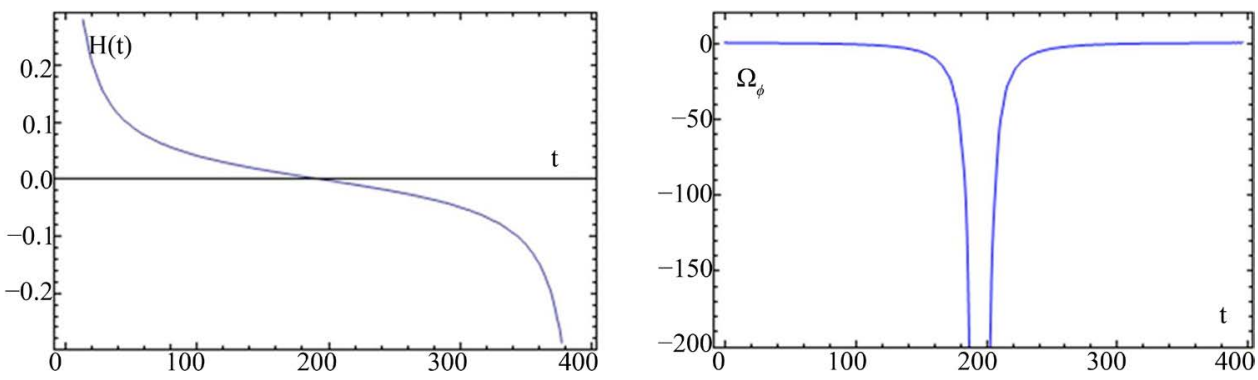

Figure 9. Left: Hubble parameter. It is a null quantity about $t \simeq 200$ approx. Right: Relative density of the field. As $H(t)$ vanish, $\Omega_{\phi}$ becomes a divergent quantity near the null point.

explained observing Equation (26). The energy evolution of a barotropic fluid $\rho_{b}$ is given by

$$
\dot{\rho}_{b}=-3 H\left(\rho_{b}+P_{b}\right)=-3 H \rho_{b}(1+w),
$$

and for a scalar field with energy density $\rho_{\phi}=E_{k}+V(\phi)$ and pressure

$$
\begin{aligned}
P_{\phi}=E_{k}-V(\phi) \text { and } E_{k}=\dot{\phi}^{2} / 2 \\
\dot{\rho}_{\phi}=-3 H\left(\rho_{\phi}+P\right)=-3 H \dot{\phi}^{2}=-6 H E_{k} .
\end{aligned}
$$



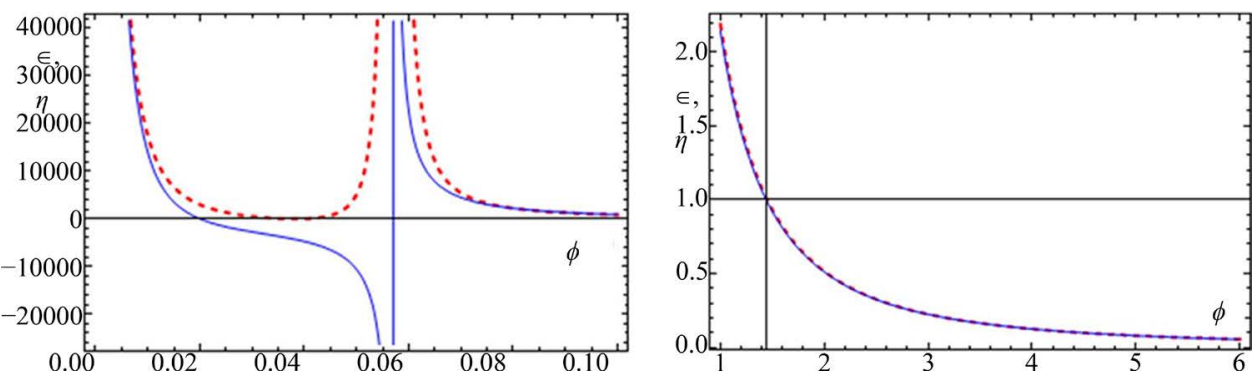

Figure 10. Slow roll parameters $\epsilon$ (dashes-red curve), and $\eta$ (continuous-blue). Left. From 0 to 0.1 in $\phi$. Right. From 1 to 6 in $\phi$. Only in the region $\phi \gtrsim 1.4$ approx. (and further on) one can expect the acceleration conditions $\epsilon \ll 1, \eta \ll 1$ to be satisfied.
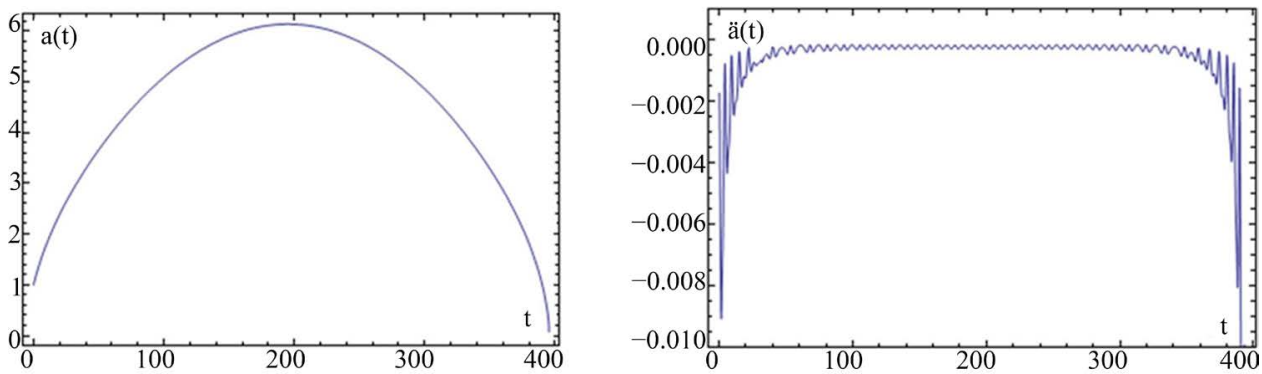

Figure 11. Left: Scale factor. Right: Acceleration $\ddot{a}(t)$. Both plots are to be interpreted as describing a universe which expands without acceleration (note that $\ddot{a}(t)$ is never greater than zero), reaching a maximum value about $t \simeq 200$ approx., thereafter falling in a contracting phase all the way long to collapse.

We can see from Equations (40) and (41) that for a positive barotropic fluid $\rho_{b}$ with an EQS $|w|<1$, the sign of $\dot{\rho}_{b}$ and $\dot{\rho}_{\phi}$ are negative as long as $H$ is positive while they become negative for $H<0$. Therefore $\rho_{b}, \rho_{\phi}$ are decreasing functions as a function of time for $H>0$ and increasing for $H<0$. Since we have seen that $\dot{H}$ is negative, this implies that $H$ is always a decreasing function of time. If $H$ can vanish at a finite time only if $\rho_{\phi}$ becomes negative, i.e. if the potential $V$ becomes negative and $E_{k}=-V$ at say $t=t_{c}$. After this time $H\left(t>t_{c}\right)$ becomes negative and will remain negative for $t>t_{c}$ and $\rho_{b}$ and $\rho_{\phi}$ will start growing with time for $t \geq t_{c}$.

Figure 8 show both kinetic and potential energies, and we can see that even though the initial kinetic energy is zero, it overtakes the potential energy and remains so until the collapsing moment $t_{\text {final }}$ when $a(t)=0$ at late times. Nevertheless, the potential energy also grows as the time is approaching $t_{\text {final }}$, so the field amplitude is eventually expelled from oscillating about the minimum.

\section{NJL Fluid with a Cosmological Constant}

Due to its theoretical properties and observational requirements, a Cosmological Constant is a very usual and useful ingredient included in cosmological models, and it is worth to consider such contribution in our model. Its defining property is an energy density $\rho_{\Lambda}$ which does not vary in time, and a coefficient of state $\omega_{\Lambda}=-1$, which 
gives a pressure $P_{\Lambda}=-\rho_{\Lambda}$. In a universe containing only a Cosmological Constant, the equation (34) is written $\ddot{a}=(8 \pi G / 3) a \times \rho_{\Lambda}$ which, as $\rho_{\Lambda}>0$, implicates $\ddot{a}(t)>0$ always. Therefore, such an universe is always accelerating its expansion. In fact, in this case the Equation (24) may be solved analytically, after substituting Equation (25), giving the well known solution $a(t)=a_{i} \exp \left(t \sqrt{8 \pi G \rho_{\Lambda} / 3}\right)$. How do the presence of a Cosmological Constant affect our previous considerations of a universe including our NJL fluid, besides matter and radiation components? Will the universe accelerate or collapse, even in the presence of a scalar field with a negative potential $V<0$ ? Because the density $\rho_{\Lambda}$ is constant, we have that the differential equations are not modified, other than just adding a term in the expression for $H$, equation (25). In particular, the equation of motion Equation (33) remains unchanged, so the field dynamics is not affected. As before, we have to deal with two cases.

a) Free Fermions $\left(g<g_{c}\right)$. As studied before, the potential is $V \geq 0$, and its minimum value is $V_{\min }=0$. Also, with the pass of time, both matter and radiation densities dilute, going to vanish. From Equation (34), it can be deduced the condition for universe to decelerate:

$$
\rho_{\Lambda}<\rho_{r}+\frac{1}{2} \rho_{m}+2 E_{k}-V(\phi) \quad(\text { for } \ddot{a}<0) .
$$

Given that the left hand side in this inequality is diminishing in time, whereas the right hand side remains constant, we have that eventually this inequality cannot hold anymore, and becomes an equality, meaning $\ddot{a}=0$. This points the beginning of the acceleration period, i.e. $\ddot{a}>0$, where the inequality (42) gets inverted. Had the initial conditions been such that inequality (42) were the opposite, then there would be always an acceleration holding always, because the LHS would never go back to grow.

Thus, we see that for a free fermions NJL fluid with a Cosmological Constant, the universe necessarily accelerate, the precise moment depending on the amount of energy densities $\rho_{m}, \rho_{r}$, with respect to that of $\rho_{\Lambda}$. This can be specified in the initial conditions, which in their turn can be chosen to solve for a realistic model fitting the observations.

b) Fermion Condensate $\left(g>g_{c}\right)$. We found before that, for a strong coupling, the potential is negative when minimized, $V_{\min }<0$. Do the universe necessarily accelerate also in this case? In order for this to happen, condition (42) eventually must turn into an equality, meaning $\ddot{a}=0$. This is a minimal condition to be satisfied, because it points at least the beginning of an acceleration; it remains to be sure that acceleration will be sustained. Let us label all quantities with a subindex " $a c$ " at time $t_{a c}$, when $\ddot{a}=0$ (vgr. $\left.V\left(t_{a c}\right)=V_{a c}\right)$. From Equation (36), we have ${ }^{6}$

$$
\rho_{\Lambda} \geq \rho_{\text {rac }}+\frac{1}{2} \rho_{\text {mac }}+2 E_{k a c}-V_{a c} \quad(\text { for } \ddot{a} \geq 0) \text {. }
$$

Remember that the potential take positive values as well as negative ones, so both possibilities must be taken into account. Certainly one can find such set of values of $V$

${ }^{6} \rho_{\Lambda}$ does not need a label because it is a constant. 
for a given $\rho_{\Lambda}$ to satisfy the inequality. However, if we rather want to consider realistic models, we should consider plausible values from observations (besides, we would not like to complicate our lives by considering unrealistic generic situations).

From definitions (30) it can be found that $\Omega_{r} / \Omega_{m}=(1+z) r$, where $z$ is the redshift, and $r=\Omega_{r 0} / \Omega_{m 0}$ says the amount of radiation with respect to that of matter. The subindex " 0 " refers to current values, i.e. quantities measured "today". Now, the estimate for $z$ (the time when acceleration begins) is around $z \sim 1$; and it has been measured $r \sim 10^{-4}$ (for the seek of simplicity, here we are interested only in orders of magnitude). Then we have $\Omega_{\text {rac }} \sim 10^{-4} \times \Omega_{\text {mac }} \ll \Omega_{\text {mac }}$, or $\rho_{\text {rac }} \ll \rho_{\text {mac }}$. Now, remember that a decelerating period dominated by matter is supposed to have taken place before $\ddot{a}=0$. In order for this to happen, condition (42) should have to be true before condition (43). For $z \sim 1$ (it could be even as big as, let's say $z \sim 10$, as this would not change the essence of the argument) and using condition (42) we would have

$$
\left.\rho_{m}>2\left(\rho_{\Lambda}+V-2 E_{k}\right) \quad \text { (in order to be } \ddot{a}<0\right) \text {. }
$$

If a positive acceleration eventually come up, the above expression is expected to become an equality. Now, suppose $V>0$. Then, unless $E_{k}$ decrease even fast, the RHS in the inequality should be decreasing as time passes, because the potential is minimizing. But $E_{k}$ cannot behave like that indeed, as the field is under a damped rolling, not to mention that $E_{k}$ is never a negative quantity, so the sum of terms $V-2 E_{k}$ will end up decreasing (would the values of these terms been such that the equality somehow would be accomplished at some time, in this case the acceleration could not be attached to $\rho_{\Lambda}$ anyway). On the other hand, for $V<0$, the inequality would become even more strong in time, because again, the potential is minimizing: $V \rightarrow V_{\min }$, and $0>V>V_{\min }$. Therefore, if initially the inequality (44) begins being satisfied, it will remain being so always; in other words, the universe will never accelerate.

What about a collapse in the future? May the presence of a cosmological constant prevent a decreasing scale factor (time going forward)? For a growing scale factor we have $\dot{a}>0$, which is true indeed because we take $H_{i}>0$ is the initial value of $H .^{7}$ As we explained before, if the scale factor is to reach a maximum $a=a_{\max }$, it must be $\dot{a}=0$. Let us name $t_{a m}$ the time when this is accomplished (if so), and label with a subindex "am" the variables valuated at this time. We have for the total energy density $\rho_{a m}=0$, thus $\rho_{\Lambda}+\rho_{\text {ram }}+\rho_{\text {mam }}+E_{\text {kam }}+V_{a m}=0$. The only way in which this could happen is for $V_{a m}<0$. In that case $V_{a m}=-\left|V_{a m}\right|$, so the equation, as a condition to be satisfied by $\rho_{\Lambda}$, can be written in the more intelligible form

$$
\rho_{\Lambda}=\left|V_{a m}\right|-E_{\text {kam }}-\rho_{\text {mam }}-\rho_{\text {ram }} \quad(\text { to get } \dot{a}=0) .
$$

If we want to keep our analysis as simple as possible, we may ignore the contribution from radiation, $\rho_{\text {ram }}=0$ (observe that, had an acceleration would be possible, then we should assume $t_{a c}<t_{a m}$, i.e. acceleration before receding, otherwise the model would not be useful. So, if $\rho_{\text {rac }} \ll 1$ the approximation $\rho_{\text {ram }} \sim 0$ is even better, as $\left.\rho_{\text {ram }}<\rho_{\text {rac }}\right)$.

${ }^{7}$ Observe this initial condition must be taken to be positive, because otherwise, the universe would be already contracting. 
Now, nothing forbids to exist a potential sufficiently deep $V_{\min }<0$, so that the equality (45) can be accomplished. The exact time at which this is achieved will depend on the relative amounts $E_{\text {kam }}, \rho_{\text {mam }}$, with respect to $\rho_{\Lambda}$, i.e. on the initial conditions. However, we can estimate a limit value by making $\rho_{\text {mam }} \rightarrow 0, E_{\text {kam }} \rightarrow 0$, and a stabilized potential $V \rightarrow V_{\min }$. Then we have

$$
\rho_{\Lambda}=\left|V_{\text {min }}\right| \quad \text { (Maximum allowed value for the universe to collapse). }
$$

After $\dot{a}=0$, i.e. $H=0$ (Equation (24)), the universe must enter into a contraction phase because $H$ is always decreasing (Equation (32)), meaning $H_{a m} \rightarrow H<0$, i.e. $\dot{a}<0$. So, eventually the universe will collapse in the future in a finite lapse of time. For $\rho_{\Lambda}>\left|V_{\text {min }}\right|$, the scale factor would never go to contract, as in this case the total energy density $\rho$ would never vanish.

It is interesting to observe that a Cosmological Constant may be seen as a particular case of a scalar field evolving under a potential stabilized with a positive minimum. As we have seen, the NJL model has two different behaviours depending on the value of the coupling constant $g$. For weak coupling $g<g_{c}$ the potential $V(\phi)$ has a minimum at the origin with $V(\phi=0)=0$ and $V(\phi) \geq 0$ otherwise. On the other hand, at strong coupling $g>g_{c}$ one has a negative minimum $\left.V(\phi)\right|_{\min }<0$. So let us approximate the potential $V$ around the minimum and take the ansatz

$$
V(\phi(t))=V_{o}+\frac{1}{2} m^{2}\left(\phi(t)-\phi_{o}\right)^{2},
$$

with $V_{o}$ a constant value (it would be $V_{o}=0$ at weak coupling and $V_{o}<0$ at strong coupling) and $\phi_{0}$ a constant. We can now ask ourself if we can have an accelerating universe. The evolution of the scalar field is just $\ddot{\phi}^{\prime}+3 H \dot{\phi}^{\prime}+m^{2} \phi^{\prime}=0$, with $\phi^{\prime} \equiv \phi-\phi_{0}$ and we could redefine

$\rho_{\Lambda}+\rho_{\phi}=\rho_{\Lambda}+E_{k}+V=\rho_{\Lambda}+V_{o}+E_{k}+\frac{1}{2} m^{2}\left(\phi-\phi_{o}\right)^{2}=\rho_{\Lambda}+V_{o}+E_{k}+\frac{1}{2} m^{2} \phi^{\prime 2}$ which corresponds to a massive scalar field with energy density $\rho_{\phi^{\prime}}=E_{k}+\frac{1}{2} m^{2} \phi^{\prime 2}$ in the presence of a cosmological constant $\rho_{\Lambda}^{\prime}=\rho_{\Lambda}+V_{o}$. A massive scalar field may accelerate the universe only at large values of $\phi^{\prime}$ (larger than the Planck mass) when the Slow Roll parameters $\epsilon$ and $\eta$ are smaller than one, while at a late time when the scalar field oscillates around the minimum the energy density $\rho_{\phi^{\prime}}$ redshifts as matter, i.e. $\rho_{\phi^{\prime}} \propto 1 / a^{3}$. In order to have $\ddot{a}>0$ we must have the quantity $\xi \equiv \rho+3 p<0$. So for a scalar field (with potential given in Equation (47)) a barotropic fluid, which we now take for simplicity as matter (without lose of generality), and a cosmological constant $\rho_{\Lambda}$, we have $\xi=\rho_{m}+4 E_{k}-2\left(\rho_{\Lambda}+V\right)$. Since the potential $V_{o}$ vanishes at weak coupling and is negative at strong coupling, there is a cancelation between the two cosmological constants $\rho_{\Lambda}$ and $V_{o}$, and the NJL model plays therefore against an accelerating phase around the minimum of the potential, since $V_{o}$ is negative.

\section{Dark Energy from NJL and SUSY Gauge Theory}

As we have seen until now, the original NJL model has interesting cosmological conse- 
quences. However, the model by itself does not reproduce the observed feature of an accelerated expansion of the universe, and it is not desirable to introduce a cosmological constant by hand, without a good explanation. We rather ask for any model to be motivated from a deeper fundamental theory. Nowadays, a paradigm for such fundamental theory is played by Super Symmetric Field Theories, and a lot of work has been done in attempting to explain Dark Matter as well as Dark Energy as some super symmetric particle (references are given in the introduction, sec. 1). Nevertheless, any conclusive theory has been established yet to present date. We would like now to generalize the NJL potential to include a physically motivated potential from supersymmetric gauge theories. These class of models have been previously studied in Dark Energy models derived from gauge theory [28] [29] (and references therein), [21] [28]-[35] and are based on ADS (Affleck-Dine-Seiberg) superpotential [35]-[38]. The derived potential is of the form

$$
U=\tilde{\Lambda}^{4+n} \phi^{-n},
$$

which is obtained from a non-perturbative super potential in a gauge theory, e.g. $n=2\left(N_{c}+N_{f}\right) /\left(N_{c}-N_{f}\right)$ for an $S U\left(N_{c}\right)$ with $N_{f}$ flavours, and $\phi$ represent a fermion condensate, i.e. $\phi \approx\langle\bar{\psi} \psi\rangle$. The condensation energy $\tilde{\Lambda}$ is the scale of breaking of the gauge symmetry. ${ }^{8}$ We now add the potential in Equation (48) to our NJL model. Since the effective NJL potential in Equation (13) has a quadratic term $\frac{1}{2} m^{2} \phi^{2}$ let us take $n=2$ in Equation (48) so that we have the symmetry under $\phi \rightarrow 1 / \phi$. Then, at some lower scale $\Lambda$, the self interaction of the field $\phi$ becomes more involved and the dynamics of the field is also governed by the effective NJL potential. By adding Equations (13) and (48), we would have the total potential

$$
V=V_{N L L}+U=\frac{1}{2} m^{2} \phi^{2}-\frac{\Lambda^{4}}{16 \pi^{2}} f(x(\phi))+\frac{\tilde{\Lambda}^{6}}{\phi^{2}} .
$$

shown in Figure 12. Of course, this is an effective theory which is plausible to the ex-

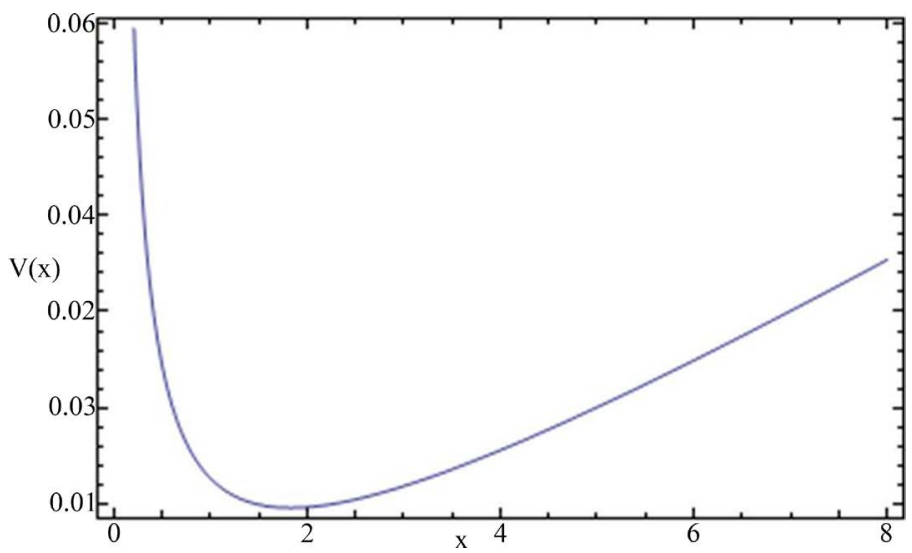

Figure 12. Graph of the total potential $V=V_{N J L}+U$, as a function of the variable $x$.

${ }^{8}$ In general, the scales $\tilde{\Lambda}$ and $\Lambda$ are not the same, and should not be confused. 
tent that NJL and Equation (48) are valid or useful theories. This is on the same footing than using the NJL model to study the dynamics of hadrons, without having obtained the model directly from the QCD Lagrangian. Since we are simply adding a term to the already studied NJL potential, we use the results of the previous section 2. Using eq. ii), (17), the condition to be satisfied by the minimum $x$ is now written (remember that we wrote before $g=s g_{c}$, Equation (20))

$$
1-\frac{1}{s^{2}}=-2 x^{2}\left(\frac{4 \pi^{2} m s \tilde{\Lambda}^{3}}{\Lambda^{4}}\right)^{2}-x \log \left(\frac{x}{1+x}\right) \equiv \alpha(x),
$$

where we have defined the function $\alpha(x)$, which is seen to be parameterized through $\tilde{\Lambda}, \Lambda, m$. This function has $-\infty<\alpha<+1$ and it is a monotonous growing function, regardless of the values of the parameters (they all are positive definite). Now, we know that the NJL potential $V_{N J L}$ is minimized in a non-trivial minimum $\phi_{\min } \neq 0$ when $g>g_{c}$, or $s>1$. In this case, the LHS in Equation (50) is a positive quantity, corresponding to $0<\alpha<1$ in the RHS, determining a solution $x=x_{0}$. This means that the total potential (49) still is minimized for some $x_{0}$, giving in its turn a non-trivial $\phi_{0}$. Given that the minimum $x_{0}$ satisfies (50), the minimized potential can be written

$$
V_{\min }=\frac{\Lambda^{4}}{16 \pi^{2}}\left[\frac{x_{0}}{s^{2}}-\log \left(1+x_{0}\right)\right]+\frac{6 \pi^{2}}{x_{0}}\left(\frac{m s \tilde{\Lambda}^{3}}{\Lambda^{2}}\right)^{2} .
$$

From this equation we see that, it is possible to obtain $V_{\min }>0$ (which would behave like a cosmological constant), if the parameters satisfy

$$
\left(\frac{m \tilde{\Lambda}^{3}}{\Lambda^{4}}\right)^{2}>\frac{1}{6\left(4 \pi^{2}\right)^{2}} \frac{1}{s^{2}}\left[x_{0} \log \left(1+x_{0}\right)-\frac{x_{0}^{2}}{s^{2}}\right] .
$$

Let us show an example. Suppose that $g=\sqrt{2} g_{c}$, i.e. $s=\sqrt{2}>1$. Also, we need to say something about the parameters, so let us take $\Lambda^{4}=8 \pi^{2} m \tilde{\Lambda}^{3}$. In this way the Equation (50) is written

$$
\frac{1}{2}=-\frac{1}{x^{2}}-x \log \left(\frac{x}{1+x}\right)
$$

which has the solution $x=x_{0} \simeq 1.83$. Then, RHS Equation (52) gives the number $x_{0} \log \left(1+x_{0}\right)-\frac{x_{0}^{2}}{2} \simeq 0.23$. This means that, in order for the potential to be positive at the minimum, the parameters must satisfy $m \tilde{\Lambda}^{3} / \Lambda^{4}>1.22 \times 10^{-5}$. We can use Equation (51) to obtain $V_{\min }$; in order of magnitude we have $V_{\min } \sim 10^{-2} \Lambda^{4}$. Let us now estimate some real physical values. The total energy density today is about $\rho_{o}=E_{o}^{4} \sim\left(10^{-3} \mathrm{eV}\right)^{4}$, and the Dark Energy contribution is $\rho_{D E o}=\Omega_{D E o} \rho_{o}$. If we identify our NJL fluid with $\mathrm{DE}$, we would have $E_{k}+V(\phi)=\rho_{D E O}$. Now, in the limit of stabilized fields about the minimum ${ }^{9}$ the energy density of our NJL fluid is $E_{k}+V(\phi) \rightarrow V_{\min }$. Then, (approximating $\Omega_{D E o} \simeq 2 / 3 \sim 1$ ) we may write $\rho_{D E o}=E_{0}^{4}=V_{\min }$. Thus we may write

${ }^{9}$ We must keep in mind that in general, the field could be in a rolling regime, so the kinetic energy would not be negligible. Therefore we must be careful in the conditions that we are talking about. 


$$
\epsilon \Lambda^{4}=10^{-3} \mathrm{eV}
$$

with the precise value of coefficient $\epsilon$ depending on the value $V_{\min }$, as shown above (for our example we have $\epsilon \sim 10^{-2}$ ). Given that this theory allows $V_{\min }>0$ as a result of the dynamics of the field, we have a possible explanation for the presence of a cosmological constant, and an accelerating universe.

\section{Summary of Results and Discussion}

The fermion model of Nambu and Jona-Lasinio (NJL) includes two different fermion states resulting from quantum effects, each one being associated with two different physical phases. For a weak coupling $g<g_{c}$ we have massless fermion fluid, whereas for a strong coupling $g>g_{c}$ a massive fermion condensate fluid is obtained. In this later case we can determine the mass of fermions and it is due to non-perturbative effects due to the strong coupling. A very convenient way to describe the system is to consider an equivalent scalar field $\phi$ moving under an effective potential $V=V_{0}^{\phi}+V_{1}^{\phi}$, which has a different form depending on the coupling strength.

Notice that in the strong coupling case $g>g_{c}$, the potential has a non-trivial negative minimum due to the negative contribution one-loop potential $V_{1}$ in Equation (6). The negative sign of this potential is due to the fermionic origin of $\psi$ field, and we have chosen to parameterize the fermion condensate in terms of an effective scalar field $\phi \sim\langle\bar{\psi} \psi\rangle$, as in Equation (4).

Here we studied the potential and solved the cosmological evolution for each fluid in presence of additional barotropic fluids (e.g. matter-dust or radiation).

For a weak coupling, we found a coefficient of state $\omega_{\phi}$ with oscillating values around zero, in such a way that the average value $\left\langle\omega_{\phi}\right\rangle=0$. Also, because the potential goes as $V \sim \phi^{2}$ near the minimum, we have that the NJL fluid in the form of free fermions dilutes as a matter. A universe containing such a fluid (with or without matter and/or radiation) will expand forever without accelerating. On the other hand, a universe containing this NJL fluid besides a cosmological constant (with or without matter and/or radiation), will eventually accelerate necessarily, expanding forever.

On the other hand, the strong coupling case (without a cosmological constant) always causes an eventually vanishing energy density. This is due to the fact that the potential is negative when minimized, and even the additional presence of matter and/or radiation does not prevent this to happen. Since the vanishing energy (which is associated with the scale factor reaching a maximum), is followed by a contracting period, this means that a fermion condensate always makes the universe collapsed. The energy density of the field $\rho_{\phi}$ vanishes a couple of times (one in the expanding phase, and the another one in the contracting phase). Because of this, some quantities $\left(\Omega_{\phi}, \omega_{\phi}\right)$ become inadequate to describe the fluid. It is important to point out the following interesting fact:

Equation (23) has been known and well studied since long time ago. If the curvature parameter is $k=+1$, the universe is said to have a spherical geometry; the scale factor is expected to get a null value eventually, so we have a collapsing universe. Because a 
spherical universe is also finite or closed, a collapsing universe was always associated with a closed universe. On the other hand, if $k=0$, the universe has a flat geometry. For ordinary matter the total energy density could be diminishing, but it could never vanish effectively in a finite time, so the scale factor in this case is expected to be always increasing. Because divergent geodesic lines in a plane never meet again, a flat universe is said to be open. So, an open universe was thought to be infinite in size (although not necessarily, but in any case, always growing). Now, remember that from the beginning, in our present study, we have taken the curvature parameter to be $k=0$, so we have been treating with a flat universe all the time. Nevertheless, we found that, if the universe contains a scalar field with a negative potential, then a future collapse cannot be avoided, giving a collapsing flat universe! In particular, because a negative potential arises naturally for the NJL model, a collapsing flat universe is also a natural consequence.

We also studied a variant of the strong coupling model, consisting in the addition of a cosmological constant. We found that, if the energy density $\rho_{\Lambda}$ is not big enough to overtake at least the minimized potential $V_{\min }$, the eventual receding of the scale factor cannot be avoided, and the universe will collapse inevitably. But if $\rho_{\Lambda}$ exceeds $V_{\min }$, then the scale factor will accelerate eventually, and the collapse will be absent.

Perhaps it is worth to emphasize that, in both cases of weak and strong coupling and without considering a cosmological constant, one may induce an acceleration of the scale factor by manipulating the initial condition for the field amplitude $\phi_{i}$, but we do not interest in it because 1) it has to be fine-tuned, and 2) it does not allow to include realistic models in which a previous deceleration period of matter dominance took place.

It is important to keep in mind that, once we settle a coupling strength (weak or strong), there is nothing in the theory to allow to switch between them, so actually a phase transition cannot be considered.

A very appealing feature of the NJL model is, in our opinion, the fact that 1 ) it is based on a "fundamental" symmetry (chiral symmetry), 2) the model leads to a potential which, due to quantum corrections, can adopt negative values in a natural way, and 3 ) it includes only one parameter: the coupling constant $g$ (two parameters if we count the cut-off $\Lambda$ ). In return we obtain interesting consequences, as allowing more than one physical phase (each having different cosmological implications), and the possibility of a collapsing universe. This is to be compared with other models involving a symmetry breaking ${ }^{10}$ or introducing new kinds of fluids aimed to be relevant to cosmological problems, but at the expense of introducing several fields or parameters. ${ }^{11}$

Finally, we saw that by considering an additional term besides the NJL potential, in

\footnotetext{
${ }^{10}$ For instance in Higgs-like models are required two parameters " $m$ " and " $\lambda$ " in order to get a potential $V=\frac{1}{2} m^{2} \varphi^{2}+\frac{1}{4} \lambda \varphi^{4}$, which have to have a "correct" relation between them in order to break the symmetry.

${ }^{11}$ For instance, to "justify" the existence of scalar fields with useful potentials, frequently one has to invoke more sophisticated theories, like String, Kaluza-Klein, GUT’s, etc. which demand a bigger effort to derive relevant results, and often implicate new exotic physics.
} 
the form of an inverse power (which is motivated from some supersymmetric theories), then it is possible to obtain a total potential with a positive minimum, thus allowing to explain a cosmological constant as a consequence of a field dynamics, which is a fermion particle (instead of a scalar field) governed by simple basic symmetries.

\section{Acknowledgements}

A.M. acknowledges financial support from UNAM PAPIIT Project No. IN101415 and Conacyt Fronteras Project No. 281.

\section{References}

[1] Perlmutter, S., et al. (1999) Astrophysical Journal, 517, 565-586. http://dx.doi.org/10.1086/307221

[2] Riess, A.G., et al. (1998) Astronomical Journal, 116, 1009-1038. http://dx.doi.org/10.1086/300499

[3] Amanullah, R., et al. (2010) Astrophysical Journal, 716, 712-738. http://dx.doi.org/10.1088/0004-637X/716/1/712

[4] Carlos, C., et al. (2010) Astronomical Journal, 139, 519-539. http://dx.doi.org/10.1088/0004-6256/139/2/519

[5] Hicken, M., et al. (2009) Astrophysical Journal, 700, 331-357. http://dx.doi.org/10.1088/0004-637X/700/1/331

[6] Riess, A.G., et al. (2007) Astrophysical Journal, 659, 98-121. http://dx.doi.org/10.1086/510378

[7] Sakharov, A.D. (1966) Soviet Physics-JETP, 22, 241.

[8] Peebles, P.J.E. and Yu, J.T. (1970) Astrophysical Journal, 162, 815-836. http://dx.doi.org/10.1086/150713

[9] Sunyaev, R.A., et al. (1970) Astrophysics and Space Science, 7, 3-19.

[10] Blake, C. and Glazebrook, K. (2003) Astrophysical Journal, 594, 665-673. http://dx.doi.org/10.1086/376983

[11] Bennett, C.L., et al. (2013) Astrophysical Journal Supplement, 208, 20. http://dx.doi.org/10.1088/0067-0049/208/2/20

[12] Ade, P.A.R., et al. (2015) arXiv:1502.01589.

[13] Reid, B.A., Chengalur, J.N., Begum, A. and Karachentsev, I.D. (2010) Monthly Notices of the Royal Astronomical Society, 404, L60-L63. http://dx.doi.org/10.1111/j.1745-3933.2010.00835.x

[14] Percival, W.J., et al. (2001) Monthly Notices of the Royal Astronomical Society, 327, 12971306. http://dx.doi.org/10.1046/j.1365-8711.2001.04827.x

[15] Tegmark, M., et al. (2001) Monthly Notices of the Royal Astronomical Society, 328, 10391063. http://dx.doi.org/10.1046/j.1365-8711.2001.04902.x

[16] Padmanabhan, N., et al. (2012) Monthly Notices of the Royal Astronomical Society, 427, 2132-2145. http://dx.doi.org/10.1111/j.1365-2966.2012.21888.x

[17] Anderson, L., et al. (2014) Monthly Notices of the Royal Astronomical Society, 441, 24-62. http://dx.doi.org/10.1093/mnras/stu523

[18] Eisenstein, D.J., et al. (2005) Astrophysical Journal, 633, 560-574. http://dx.doi.org/10.1086/466512 
[19] Tojeiro, R., et al. (2014) Monthly Notices of the Royal Astronomical Society, 440, 22222237. http://dx.doi.org/10.1093/mnras/stu371

[20] Copeland, E.J., Sami, M. and Tsujikawa, S. (2006) International Journal of Modern Physics $D, 15,1753-1935$. http://dx.doi.org/10.1142/S021827180600942X

[21] Ratra, B. and Peebles, P.J.E. (1988) Physical Review D, 37, 3406. http://dx.doi.org/10.1103/PhysRevD.37.3406

[22] Steinhardt, P.J., Wang, L. and Zlatev, I. (1999) Physical Review Letters, 82, 896. http://dx.doi.org/10.1103/PhysRevLett.82.896

[23] Steinhardt, P.J., Wang, L. and Zlatev, I. (1999) Physical Review D, 59, Article ID: 123504. http://dx.doi.org/10.1103/PhysRevD.59.123504

[24] De la Macorra, A. and Piccinelli, G. (2000) Physical Review D, 61, Article ID: 123503.

[25] De la Macorra, A. and Stephan-Otto, C. (2002) Physical Review D, 65, Article ID: 083520. http://dx.doi.org/10.1103/PhysRevD.65.083520

[26] Das, S., Corasaniti, P.S. and Khoury, J. (2006) Physical Review D, 73, Article ID: 083509.

[27] De la Macorra, A. (2007) Physical Review D, 76, Article ID: 027301. http://dx.doi.org/10.1103/PhysRevD.76.027301

[28] De la Macorra, A. (2008) Journal of Cosmology and Astroparticle Physics, 0801, 030.

[29] De la Macorra, A. (2007) Astroparticle Physics, 28, 196-204. http://dx.doi.org/10.1016/j.astropartphys.2007.05.005

[30] De la Macorra, A. (2004) Physics Letters B, 585, 17-23. http://dx.doi.org/10.1016/j.physletb.2004.02.006

[31] De la Macorra, A. (2010) Astroparticle Physics, 33, 195-200. http://dx.doi.org/10.1016/j.astropartphys.2010.01.009

[32] De la Macorra, A. (2005) Physical Review D, 72, Article ID: 043508.

[33] De la Macorra, A. (2003) Journal of High Energy Physics, 0301, 033.

[34] De la Macorra, A. and Stephan-Otto, C. (2001) Physical Review Letters, 87, Article ID: 271301. http://dx.doi.org/10.1103/PhysRevLett.87.271301

[35] Binetruy, P. (1999) Physical Review D, 60, Article ID: 063502. http://dx.doi.org/10.1103/PhysRevD.60.063502

[36] Masiero, A., Pietroni, M. and Rosati, F. (2000) Physical Review D, 61, Article ID: 023509.

[37] Lyth, D.H. and Riotto, A. (1999) Physics Reports, 314, 1-146. http://dx.doi.org/10.1016/S0370-1573(98)00128-8

[38] Affleck, I., Dine, M. and Seiberg, N. (1985) Nuclear Physics B, 256, 557-599. http://dx.doi.org/10.1016/0550-3213(85)90408-0 
Submit or recommend next manuscript to SCIRP and we will provide best service for you:

Accepting pre-submission inquiries through Email, Facebook, LinkedIn, Twitter, etc.

A wide selection of journals (inclusive of 9 subjects, more than 200 journals)

Providing 24-hour high-quality service

User-friendly online submission system

Fair and swift peer-review system

Efficient typesetting and proofreading procedure

Display of the result of downloads and visits, as well as the number of cited articles

Maximum dissemination of your research work

Submit your manuscript at: http://papersubmission.scirp.org/

Or contact jmp@scirp.org 